\title{
Processing of High-Frequency Multibeam Echo Sounder Data for Seafloor Characterization
}

\author{
Laurent Hellequin, Jean-Marc Boucher, Member, IEEE, and Xavier Lurton
}

\begin{abstract}
Processing simultaneous bathymetry and backscatter data, multibeam echosounders (MBESs) show promising abilities for remote seafloor characterization. High-frequency MBESs provide a good horizontal resolution, making it possible to distinguish fine details at the water-seafloor interface. However, in order to accurately measure the seafloor influence on the backscattered energy, the recorded sonar data must first be processed and cleared of various artifacts generated by the sonar system itself. Such a preprocessing correction procedure along with the assessment of its validity limits is presented here and applied to a $95-\mathrm{kHz}$ MBES (Simrad EM1000) data set. Beam pattern effects, uneven array sensitivities, and inaccurate normalization of the ensonified area are removed to make possible further quantitative analysis of the corrected backscatter images. Unlike low-frequency data where the average backscattered energy proves to be the only relevant feature for discriminating the nature of the seafloor, high-frequency MBES backscatter images exhibit visible texture patterns. This additional information involves different statistical distributions of the backscattered amplitudes obtained from various seafloor types. Non-Rayleigh statistics such as $\boldsymbol{K}$-distributions are shown to fit correctly the skewed distributions of experimental high-frequency data. Apart from the effect of the seafloor micro-roughness, a statistical model makes clear a correlation between the amplitude statistical distributions and the signal incidence angle made available by MBES bathymetric abilities. Moreover, the model enhances the effect of the first derivative of the seafloor backscattering strength upon statistical distributions near the nadir and at high incidence angles. The whole correction and analysis process is finally applied to a Simrad EM 1000 data set.
\end{abstract}

Index Terms-Backscatter model, $K$-distribution, multibeam echo sounder (MBES), seafloor classification.

\section{INTRODUCTION}

$\mathbf{M}$ ANY marine activities (marine geology, commercial fishing, offshore oil prospecting and drilling, cable and pipeline laying and maintenance, and underwater warfare) need tools and methods to remotely characterize the seafloor. Modern swath-mapping sonars are well designed for this task; they have quickly evolved upwards over the last 40 years and nowadays are beginning to meet most of the requirements needed to reliably characterize the seafloor. Among the existing acoustical mapping systems, multibeam echo sounders (MBESs) are currently the main focus of attention because of their ability to provide both a bathymetric map and a backscatter image of the surveyed area.

Manuscript received xxxx; revised xxxx. [AUTHOR: PLEASE PROVIDE THE MANUSCRIPT RECEIVED AND REVISED DATES.-ED.]

L. Hellequin and X. Lurton are with IFREMER, TMSI/AS, Technopôle Iroise, BP 70, 29280 Plouzané, France.

J. M. Boucher is with ENST Bretagne, BP 832, 29285 Brest Cedex, France. Digital Object Identifier 10.1109/JOE.2002.808205
Usually installed under a ship's hull, an MBES transmits a sound pulse inside a wide across-track and narrow along-track angular sector; then a beamforming process simultaneously creates numerous receiving beams steered at different across-track directions. This spatial filtering allows us to pick up echoes coming from adjacent seafloor portions independently. One sounding is accurately computed inside each beam by simultaneously measuring the beam steering angle and the echo travel time, according to various estimation methods based on either amplitude or phase. A high density of sounding points is thus generated along the survey swath, and new "pings" are transmitted as the ship proceeds on her way. Taking into account the ship's navigation and attitude, the data from successive pings are finally gridded together in order to create an accurately georeferenced digital terrain model (DTM).

In addition to measuring the echo travel times and angles for bathymetry, an MBES also records the echo amplitudes containing information about the nature and geoacoustical properties of the seafloor. The echo amplitude is typically remapped to a color or gray scale and forms a coregistered backscatter image. The short pulse length provides the high resolution needed for imaging seafloor backscatter with a sufficient amount of details.

For low-resolution MBESs (working in deep water at lower frequencies, typically $12 \mathrm{kHz}[1]$ ), it seems that the mean backscattering strength (BS), recorded as a function of the incident angle, is the only measured parameter usable to characterize the interface acoustical properties [2]. However, for MBESs with better resolution (designed for shallow depths with higher frequencies, typically $100 \mathrm{kHz}$ [3]), more information is available from the backscattered signals for a better seafloor characterization.

A typical example of a BS image with a good resolution (Fig. 1) shows various textures and spatial organizations of pixels that are clearly related to variations in the nature of the seafloor. In addition to its average level, the BS variability within subareas makes it possible to improve seafloor characterization using statistical techniques [4], [5]. Better classification results are expected when the MBES characteristics (frequency, beamwidth, and incidence angle) and an appropriate BS model are used to refine the analyses.

Analyzing a backscatter image in detail reveals several artifacts that degrade the image and corrupt BS measurements. The strong specular echo, causing a high-level line under the ship's track, is linked to the backscattering physics and is not to be considered, properly speaking, as an artifact; however, it is a penalizing feature, quite difficult to erase from sonar images. The main artifact comes from the directivity patterns of arrays used for the signal transmission and reception, that are usually not 


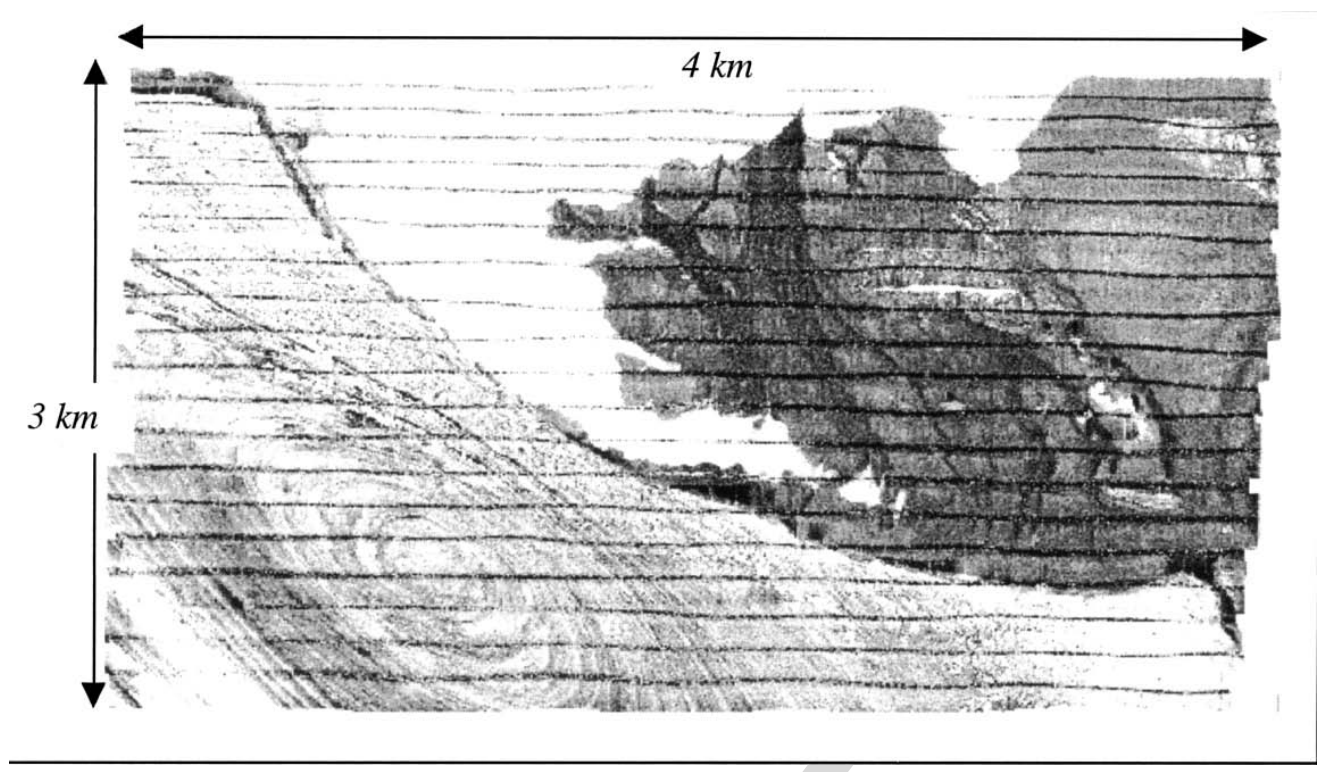

Fig. 1. Backscatter image recorded with a high-frequency MBES on the French inner shelf [6]. The along-track stripes correspond to the strong echo level at the nadir.

accurately compensated for in the time series processed by the sonar; this results in parallel lines along the ship's track, that are commonly observed for many systems. Another noticeable artifact related to the echo-sounder may be due to the time-varying gain (TVG) function, designed to attenuate the backscattered level from the specular direction and to increase it at high incidences; problems arise if its computation is not well adapted to the physics of the measurement configuration.

Depending on the MBES characteristics, these local amplitude perturbations may, in some cases, greatly exceed the permitted amplitude sensibility (typically $2-3 \mathrm{~dB}$ ) needed to discriminate two different seafloors by their average BS. These artifacts must be eliminated during preprocessing by applying adequate correction procedures implying the estimation of the MBES characteristics involved. The aim of this preprocessing is to use the backscattered signals to derive new data independent of the acquisition system and dependent only on the seafloor properties, with a view to further amplitude statistics processing. These preprocessing techniques, described in Section II of this paper, can be run efficiently on any MBES data set since the bathymetry and BS data are coregistered. Simulations of the sonar receiving process eventually stress the limits of validity of the correction procedure when reception beams are steered very close to the nadir.

After this description of operations, Section III discusses the statistical analysis of backscattered amplitudes recorded by MBES systems. It is shown that characterization potentialities can be recovered from the statistical distributions of the backscatter amplitude. In particular, the classical Rayleigh distribution is not well adapted to rough seafloor data, while non-Rayleigh statistics such as $K$-distributions correctly fit the skewed distributions obtained in some data from sedimentary areas, thus providing new parameters for characterization. It is also shown that the $K$-distribution depends on the incident angle. These theoretical results are combined to propose the hypothesis of a correlation between the backscatter amplitude statistical distribution and the first angular derivative of BS. Moreover, MBES geometry is used to emphasize the correlation of BS statistics with the incident angle in addition to the ensonified area size.

\section{PREPROCESSING AND SIGNAL CORRECTIONS}

\section{A. Description of the MBES Artifacts}

Various simplifications are often to be found in real-time processing software used for MBES image processing (e.g., no compensation for residual beam directivity patterns and over-simplified footprint size compensation). These approximations may render the statistical analysis difficult-if not pointless - since they strongly affect the statistical distribution of the available backscattered data. Fig. 2 presents a sonar image of a flat homogeneous seafloor obtained with a Simrad EM 1000 (a widely used shallow-water MBES, forming 60 simultaneous beams over a $150^{\circ} \times 3.3^{\circ}$ angle sector, the frequency is $95 \mathrm{kHz}$, individual receive beams are $3.3^{\circ}$ wide in both directions, the transmit-receive array is half-cylindrical, and the pulse duration is $0.2 \mathrm{~ms}$ ). Fig. 2 also features a plot of the raw average BS versus the transmission angle. The slow and smooth gray level modulations appearing away from the near-nadir zone presumably result from uneven transducer sensitivities and electronic gains. Deeper striping, whose amplitude increases as the transmission angle goes to zero, creates strong track-parallel artifacts on the sonar image; it is obviously correlated with the beam patterns of the 60 receiving beams [6]. Finally, BS is expected to be at its highest in the near-specular zone, while the recorded level is actually at its lowest. These latter effects are due to incorrect normalization procedures used for footprint size compensations in this specular zone.

The aim of the preprocessing operations is therefore to remove these system artifacts efficiently from the MBES data without requiring an in-tank or at-sea calibration. This can be done from field data acquisition, the main requirement being 


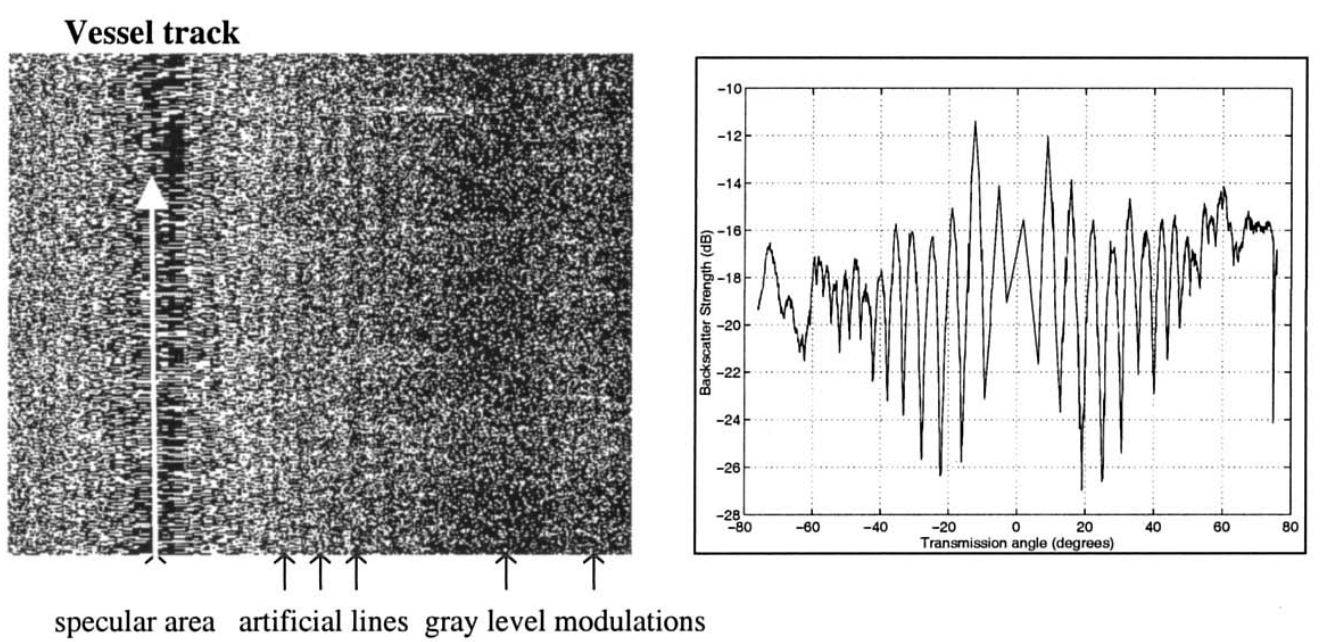

Fig. 2. Partial BS image recorded on a flat homogeneous seafloor and corresponding average BS versus the transmission angle, showing artifacts due to the array beam pattern.

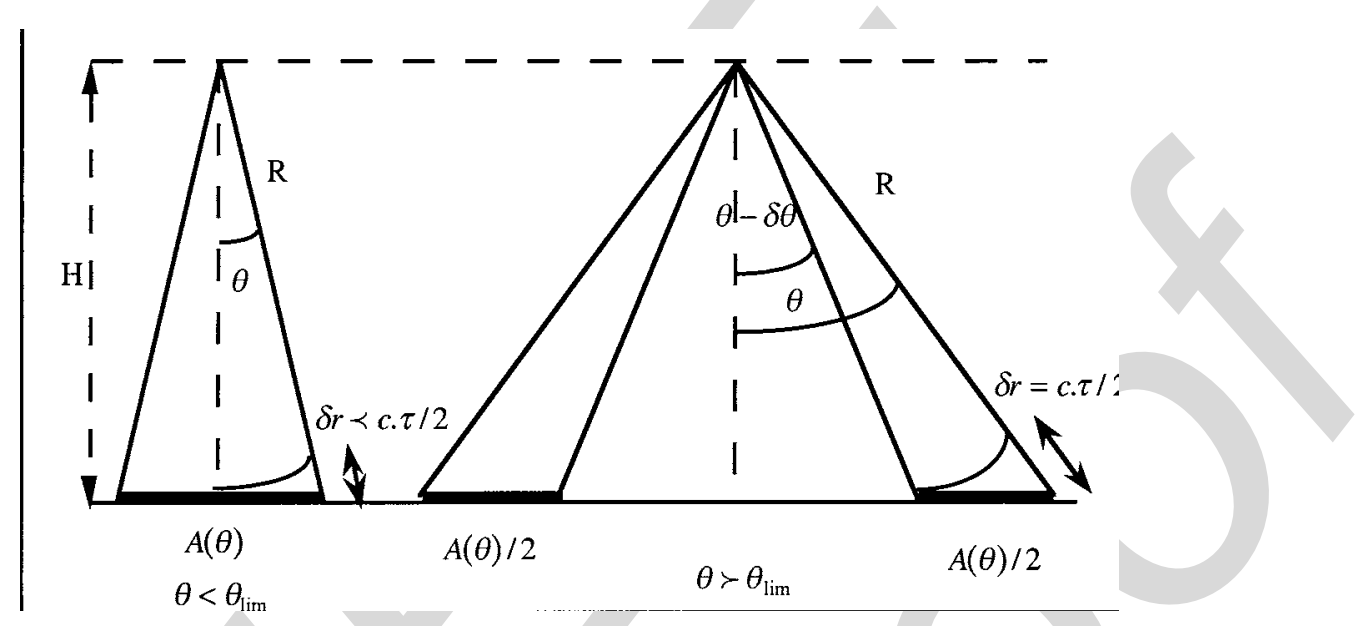

Fig. 3. The ensonified areas for the near-nadir case $\left(\theta<\theta_{\text {lim }}\right)$ and at oblique transmission angles $\left(\theta>\theta_{\text {lim }}\right)$.

that the calibration is performed over a flat homogeneous seafloor large enough to ensure good reliability of the beam pattern identification.

\section{B. Backscattering Strength Measurement}

In order to characterize the artifacts due to the echosounder, simulations of BS measurements were conducted using various parameters of the MBES and the measurement configuration (e.g., water depth, pulse time length, and beam patterns). This is also an important step prior to the definition of relevant preprocessing. For this purpose, we assume a flat seafloor and no in-water refraction effect, i.e., the transmitted and incident angle are the same.

The recorded backscattered intensity depends upon the MBES acquisition parameters in quite a complicated way. If $E L(\theta)$ (in decibels) is the received echo level, then one gets from the sonar equation

$$
\begin{aligned}
E L(\theta)=S L(\theta)- & 2 . T L(\theta) \\
& +10 \log \iint_{A(\theta)} B S_{\text {nat }}(\alpha) W^{2}(\alpha) \cdot d A(\alpha)
\end{aligned}
$$

where $\theta=a \cos (2 H / c t)$ is the transmitted incident angle, $H, c$, and $t$ are the water depth, the sound velocity, and time, respectively, $S L(\theta)$ (in decibels) is the source transmission level at angle $\theta, 2 T L$ (in decibels) is the propagation loss, and $B S_{\text {nat }}(\alpha)=10^{B S(\alpha) / 10}$ and $W^{2}(\alpha)$ are the backscattering strength and the array directivity pattern at angle $\alpha$, respectively, in natural units, to be integrated over the ensonified area $A(\theta)$.

In the near-nadir case (Fig. 3), the pulse length is greater than $R(\theta)-H$, which means that the ensonified area has to be expressed by

$$
A(\theta)=2 H \cdot \tan \theta \cdot R \cdot \theta_{a}
$$

where $\theta_{a}$ stands for the along-track beam aperture. Beyond the limit angle $\theta_{\text {lim }}=a \cos [H /(H+(c \tau / 2))]$ corresponding to $R(\theta)-H=c \tau / 2$, the ensonified area is given by

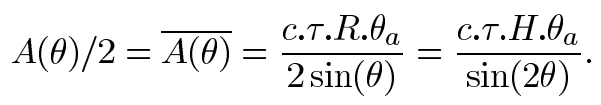

Unfortunately, (1) does not lead to a simple derivation of $B S_{\text {nat }}(\theta)$, because of the coupling inside the integral with terms 


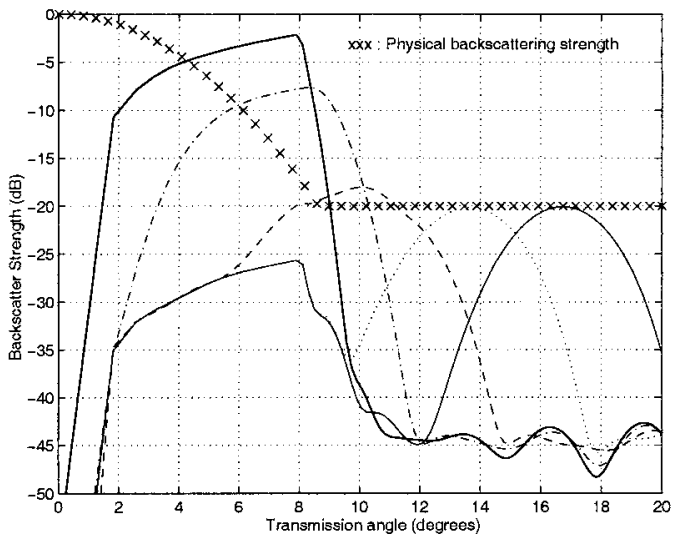

Fig. 4. Simulation of the angular backscattered level simulated in several beams. Water depth $=15 \mathrm{~m}$. Beams steering angles: (-) $1.8^{\circ},(-.-) 5.4^{\circ},(---)$ $8.9^{\circ},(\ldots) 12.3^{\circ}$, and $(-) 15.7^{\circ}$ (with respect to the nadir).

$W^{2}(\theta)$ and $A(\theta)$ linked to the sounder geometry. A common and convenient approximation is that the ensonified area $A(\theta)$ is sufficiently small for the backscattering strength to be considered constant over the integration domain; in addition, the array directivity pattern $W^{2}(\theta)$ is set constant equal to 1 . The measured backscattering strength $\overline{B S(\theta)}$ may then be directly derived from the measured echo level $E L(\theta)$ as

$$
\overline{B S(\theta)}=E L(\theta)-S L(\theta)+2 T L-10 \log \overline{A(\theta)}
$$

where $\overline{A(\theta)}$, given by (3), is the ensonified area used in the real-time algorithms.

However, these classical approximations lead to a bias between the measured value $\overline{B S(\theta)}$ and the actual physical value $B S(\theta)$ of the backscattering strength

$\overline{B S(\theta)}=10 \log \iint_{A(\theta)} B S_{\text {nat }}(\theta) \cdot W^{2}(\theta, \varphi) d A-10 \log (\overline{A(\theta)})$.

The following numerical simulations show the importance of the bias as a function of the geometrical parameters of a given MBES (water depth, pulse time duration, beam width, and transmission angle).

\section{Echo Signal Simulations}

The angular response according to (5) was computed for five beams, steered at $1.8^{\circ}, 5.4^{\circ}, 8.9^{\circ}, 12.3^{\circ}$, and $15.7^{\circ}$ with respect to the nadir. The directivity diagram $W^{2}(\theta)$ follows a Dolph-Chebyshev shading law, with a side lobe level fixed at -25 dB. Fig. 4 shows a simulation conducted with the actual MBES parameters [3] and depicts the measured backscattering strength $\overline{B S(\theta)}$ for the five beams according to (5).

These simulations show several phenomena:

- For the outermost beams $\left(12.3^{\circ}\right.$ and $\left.15.7^{\circ}\right)$, the smooth and symmetrical main lobe of the measured backscattering strength $\overline{B S(\theta)}$ can be fitted to the beam pattern shape computed with the Dolph-Chebyshev shading. The actual ensonified area $A(\theta)$ is then limited by the pulse length and equals the estimated area $\overline{A(\theta)}$ given by (3). Furthermore, the integrand in the integral term may be considered as constant over the narrow area $A(\theta)$. So (5) could be simplified into

$$
\overline{B S(\theta)}-B S(\theta)=10 \log W^{2}\left(\theta_{0}\right)
$$

with $\theta_{0}$ defined by

$$
\cos \theta_{0}=\cos \theta \frac{1}{1-\frac{c \tau \cos \theta}{4 H}} .
$$

Except for the small angular shift between $\theta$ and $\theta_{0}$ (given in Table I), no deformation actually perturbs the beam pattern $W^{2}(\theta)$ ). This justifies the preprocessing correction procedure for the off-specular transmission angles that is discussed below (see Section II-D).

- At steepest transmission angles, the measured $\overline{B S(\theta)}$ decreases. Close to nadir, the ensonified area $A(\theta)$ is limited by the across-track beam aperture and becomes smaller than its estimate $\overline{A(\theta)}$ which remains pulse-limited in the across-track plan. To compensate for this difference in area size and to retrieve a correct backscattered level at the beam central sample, $\overline{B S(\theta)}$ has to be increased by the correction term

$$
D=-10 \log \left(\frac{\theta_{T}}{c \tau} H \tan \theta\right)
$$

where $\theta_{T}$ is the $-3-\mathrm{dB}$ aperture angle of the antenna beam pattern.

Moreover, the simplification of the integral term in (1) no longer reduces to (6) in this specular area. The actual area $A(\theta)$ is not small enough for a linear dependence between $\overline{B S(\theta)}$ and the beam pattern $W^{2}(\theta)$. For the three steepest beams, $\overline{B S(\theta)}$ now derives from deformations of the Dolph-Chebyshev beam pattern as depicted in Fig. 4, which cannot be modeled analytically in the general case. Since these deformations also depend on the actual angular backscattering strength $B S(\theta)$, no efficient preprocessing correction procedure can be undertaken for these central beams. Furthermore, since the maximum backscattering level no longer fits the beam center sample, the depth determination (based upon the search for the envelope barycenter of the time signal) might be slightly biased within these specular beams [8].

\section{Artifact Preprocessing Correction Procedure for Multibeam Sonar Images}

The area correction proposed in (7) increases the backscattered signal level estimation to a more satisfactory value for the near-specular angles. Compensation for the directivity effects requires preliminary knowledge of the antenna beam pattern. In our study, since most of the directivity information was unavailable, antenna beam patterns had to be estimated from backscatter images recorded on a training zone, chosen as flat and as homogeneous as possible; the basic principle is that the directivity effect can be estimated as the difference between the actually measured $\overline{B S(\theta)}$ and a physically ideal $B S_{\phi}(\theta)$. The difficulty of the method lies in the definition of a reasonable a priori estimate of the actual backscattering strength. We chose to start from a generic angular form of BS, with sufficient generality to justify the relevance of the method a posteriori by confronting the directivity estimations obtained from several 
TABLE I

SHIFT BETWEEN $\theta$ AND $\theta_{0}(H=15 \mathrm{~m})$

\begin{tabular}{|c|c|c|c|c|c|c|}
\cline { 2 - 7 } \multicolumn{1}{c|}{} & $\begin{array}{c}\text { Exponential } \\
\text { (Rayleigh) }\end{array}$ & $\begin{array}{c}\Gamma \\
\text { distribution }\end{array}$ & Pearson system & $K$ distribution & $D_{a c c}$ & $M$ \\
\hline Rock & $73 \times 10^{-3}$ & $24 \times 10^{-3}$ & $17 \times 10^{-3}$ & $23 \times 10^{-3}$ & $7 \times 10^{-3}$ & $6 \times 10^{4}$ \\
\hline Gravel & $28 \times 10^{-3}$ & $11 \times 10^{-3}$ & $8 \times 10^{-3}$ & $7.4 \times 10^{-3}$ & $7 \times 10^{-3}$ & $6 \times 10^{4}$ \\
\hline Silt & $10 \times 10^{-3}$ & $21 \times 10^{-3}$ & $18 \times 10^{-3}$ & $4.4 \times 10^{-3}$ & $4.5 \times 10^{-3}$ & $1.2 \times 10^{5}$ \\
\hline
\end{tabular}

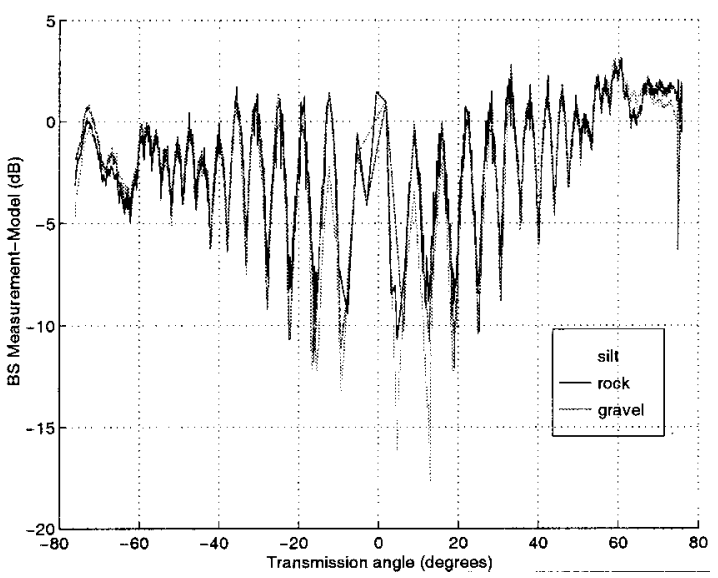

Fig. 5. Retrieval of the array directivity pattern $\overline{W^{2}(\theta)}=\overline{B S(\theta)}-B S_{f}(\theta)$ from the backscatter measurements for three different seafloors (silt, sand, and rock).

different seafloor zones. This functional physical model, noted $B S_{\phi}(\theta)$, is derived from the tangent plane model [9] (Kirchhoff approximation at high frequencies) at near nadir and is taken to be Lambert-like for off-specular angles

$$
B S_{\phi}(\theta)=10 \log \left(A \exp \left(-\alpha \theta^{2}\right)+B \cos ^{\beta} \theta\right) .
$$

Then the beam pattern estimation procedure and correction process needs to be performed in several steps.

- The angle correction (TVG) applied in the echo sounder software has to be removed in order to recover a more correct $B S(\theta)$ (this TVG correction is used in most MBESs to limit the received signal dynamics and to flatten the recorded level prior to constructing the backscatter image).

- The backscatter level of each beam center is not affected by the directivity effects, except for near-specular beams. The heuristic $B S_{\phi}(\theta)$ model is hence fitted to the level measured at these points in order to identify the coarse evolution of the backscatter, prior to its subtraction from the measured $\overline{B S(\theta)}$

- Within the range of valid values (Section II-C), $\overline{B S(\theta)}-$ $B S_{\phi}(\theta)$ should then be independent of the seafloor characteristics and reflect only the array beam pattern $10 \log W^{2}(\theta)$.

The process was run on images from three different seafloor types (silt, gravel, rock) (Fig. 5); in every case, the values $A$, $B, \alpha$, and $\beta$ were estimated by a least-mean-square error algorithm. Ignoring the three central beams, it is seen that the variations from one seafloor type to another do not exceed 1 $\mathrm{dB}$ (compared to the 10-dB dynamics of the directivity pattern oscillation, see Fig. 2). This result confirms the validity of the method a posteriori and makes us confident that the BS influ-

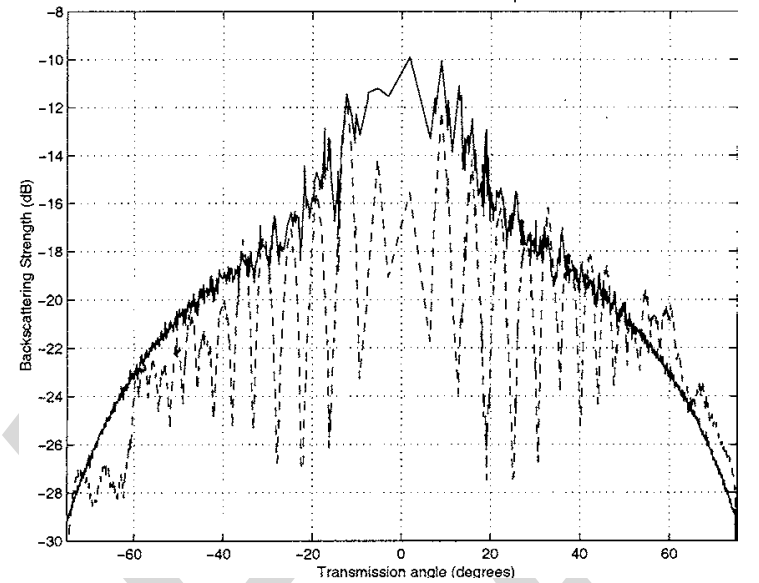

Fig. 6. Average BS level versus transmission angle before (dashed line) and after (solid line) array directivity pattern compensation.

ence of the seafloor has been successfully eliminated from the recorded signal.

- Finally, a realistic parameterized array directivity pattern model $W^{2}(\theta)$ (taking into account the array shape and shading and the beam widths and steering directions) has to be fitted to the estimated $\overline{W^{2}(\theta)}$.

- Given the beam number, the transmission angle for each pixel, and now the directivity pattern model $W^{2}(\theta)$, the corrected backscattering strength $B S_{c}(\theta)$ may readily be obtained from the transformation

$$
B S_{c}(\theta)=\overline{B S(\theta)}-10 \log W^{2}(\theta)
$$

to be applied to the entire sonar image in order to eliminate the echosounder artifacts. Note in Fig. 6 that the resulting $B S(\theta)$ curve is now symmetrical.

An easy way to implement the method is to neglect the angular shift between $\theta$ and $\theta_{0}$ both during the beam pattern identification on the training zone and during the correction procedure on different sonar image areas. However, it should be noted that the shift between $\theta$ and $\theta_{0}$ is water-depth-dependent. Hence, when correcting images from areas with depths different from the training zone, beam patterns may be slightly shifted from their actual angular position, and small residual oscillations (2 or $3 \mathrm{~dB}$ peak to peak) are prone to remain. Moreover, it should be noted that the BS level measured in the specular area may be underestimated in shallow water: if the sampling frequency is too low, the time signals from the near-specular beams may give only one or two samples; these recorded samples are not likely to correspond to the beam center instant, and an irreversible underestimation of the level may result on the recorded signal. 


\section{E. Summary of Preprocessing Corrections for Multibeam Sonar Images}

Since the BS levels recorded by MBES systems are prone to be modulated by several artifacts (mainly directivity patterns linked to array sensitivity and beamforming, and ensonified area compensation), relevant corrective preprocessing must be applied in order to make them usable for any characterization tasks. We show typical effects of these artifacts, and propose adapted corrections:

- The directivity modulations may be accounted for by estimating the resulting transmission-reception patterns extracted from experimental data. This can be done on sonar images from flat horizontal homogeneous zones, after elimination of the gross angular variations imposed by the physical BS; the latter is estimated from a simple functional model giving sufficient flexibility to fit a large variety of seafloor backscatter responses. Finally, the experimental directivity pattern has to be fitted to a parameterized model; this will be used for the final equalization of the sonar image level.

- The level adjustments computed inside the echosounder receiver (TVG law, footprint size corrections) have to be compensated and then replaced by more accurate expressions. This part of the process may be made difficult by the fact that the inner processing details of the sounder may be unavailable. It is especially important for data obtained close to the vertical, for which it is shown that the commonly admitted approximations may lead to significant errors; while this central part of the swath is of secondary importance in side-scan sonar image processing, it can hardly be neglected in MBES cartography, for which the swept angle range is much narrower.

Although this methodology was initially developed for a system for which the above problems appeared especially noticeable, the issues developed here are likely to be met for any MBES; the above-suggested correction methods may be applied as well. Although the current user's purpose is usually not to go into detailed analyses of statistical amplitudes, systematically applying these compensations proves to be of great benefit for improving the quality of sonar images, even for a qualitative interpretation.

\section{Statistical Characterization of Multibeam ECHO-SOUNDER DATA}

\section{A. Data Statistical Analysis for Seafloor Characterization}

With low-frequency MBESs used in deep water, the only useful parameter for seafloor characterization turns out to be the angle dependence of the average backscattering strength, due to the poor spatial resolution [2] caused by the wide footprint. The number of elementary scatterers in the ensonified area (large compared to the characteristic length of the seafloor roughness) becomes sufficiently high to validate the hypothesis of the central limit theorem [10]. The coherent summation of the scatterer contribution in phase and amplitude produces a Gaussian process for the complex envelope or a Rayleigh distribution for the measured amplitude.
With shorter pulse lengths, the instant ensonified area (resolution cell) may be small enough to track some small-scale seafloor characters (such as shellfish patches, local slopes, and roughness changes) that create visible nonhomogeneous zones in the sonar image (Fig. 1). Even with a large number of scatterers, the spatial variability leads to a product model [10] that describes the backscatter amplitude $K$-distribution [12] as a Rayleigh-fluctuating process modulated by a two-parameter (mean and shape factor) $\Gamma$-distributed local reflectivity representing the relief particularities [13]. This product model helps to interpret statistical changes according to the sounder geometry [14], or to seafloor characteristics [4]. In addition to this interesting model, $K$-distributions are also attractive because of their noticeable ability to fit large sets of statistical distributions on measured data collected during high-resolution coherent reverberation processes (sea surface [11], [12] or ground surface [15] radar imagery or seafloor sonar imagery [16], [4], [5]).

In this section, amplitude statistical distributions of high-frequency MBES data are analyzed. This data combines imagery and bathymetry, and hence, makes it possible to study the influence of the ensonified area, and more specifically the incidence angle, on the amplitude statistics. To interpret statistical observations according to the MBES geometry and to the relief characteristics, a $K$-distribution reverberation model [15] is used, including the relief correlation length, the ensonified area, and the backscattering strength effects.

\section{B. Statistical Models for Correlated Non-Rayleigh Reverberation Processes}

1) Scattering Point Models: In a scattering point model [10], the seafloor is modeled by a large set of discrete scatterers, each of them reflecting a randomly attenuated and delayed copy of the emitted sound pulse $s(t)$ to generate the reflected complex echo $F(\vec{r}, t)$ as

$$
F(\vec{r}, t)=\sum_{i=1}^{N} a_{i} \exp \left(j \phi_{i}\right) . s(t) .
$$

To simplify notations, the time dependence is suppressed in the following sections. $\left\{a_{i}\right\}$ and $\left\{\phi_{i}\right\}$ are the random elementary amplitudes and phase, and $N$ is the number of scatterers inside the resolution cell.

The phases $\left\{\phi_{i}\right\}$ are usually considered to be independent and uniformly distributed over $[-\pi,+\pi]$. The limit theories consider that the amplitudes $\left\{a_{i}\right\}$ are mutually independent and that $N \rightarrow \infty$. Hence, the statistical limit distributions for the normalized energy $Y=\|F\|^{2} / A\left(\theta_{\text {inc }}(\vec{r})\right)$ depend only on the random variable $N$. The validity of the central limit theorem leads to a complex Gaussian distribution for $F$, a Rayleigh distribution for the normalized amplitude $\sqrt{Y}$, and an exponential distribution for the normalized energy $Y$

$$
p_{Y}(y)=\frac{1}{\langle Y\rangle} \exp \left(-\frac{y}{\langle Y\rangle}\right) .
$$


A normalized random variable $Z$, called the speckle factor, allows the energy $Y$ to be defined according to a product model

$$
\begin{aligned}
Y & =\langle Y\rangle \cdot Z \\
p_{Z}(z) & =\exp (-z), \quad \text { with }\langle Z\rangle=1 \quad \text { and } \quad\left\langle Z^{2}\right\rangle=2 .
\end{aligned}
$$

The speckle noise is considered as a rapidly fluctuating, spatially and temporally independent process. In non-Rayleigh approaches, $\operatorname{var}(N) /\langle N\rangle^{2}$ remains constant when $N$ is large. A negative binomial distribution for $N$ fulfills this assumption [12]. This condition makes it possible to consider bunches of scatterers along moving resolution cells. The ensonified area is small enough to describe and follow the macro-relief variations. However, the validity of the central limit theorem diminishes. The negative binomial distribution for $N$ leads to a $K$-distribution for $Y$ of

$$
\begin{gathered}
p_{Y}(y)=\frac{2}{\Gamma\left(\alpha_{\mathrm{eff}}\right) \cdot y}(\beta . y)^{\left(\alpha_{\mathrm{eff}}+1\right) / 2} K_{\alpha_{\mathrm{eff}}-1}(2 \sqrt{\beta . y}) \\
\beta=\frac{\alpha_{\mathrm{eff}}}{\langle Y\rangle} \quad \alpha_{\mathrm{eff}}=\frac{1}{\frac{1}{2} \frac{\left\langle Y^{2}\right\rangle}{\langle Y\rangle^{2}}-1} .
\end{gathered}
$$

In addition to the average value, a shape factor $\alpha_{\text {eff }}$ characterizes the $Y$ statistical distribution. For great values of $\alpha_{\text {eff }}$ the $K$-distribution tends toward the exponential distribution. $K_{\alpha_{\text {eff }}-1}$ is the modified Bessel function of second kind and order $\alpha_{\text {eff }}-1$. The product model in (12) must be adapted by considering a local reflectivity $S$ [11]. The low fluctuating $\Gamma$-distributed random variable $S$ represents the influence of the macro-roughness modifying the speckle $Z$ local properties

$$
\begin{aligned}
Y & =S . Z \\
p_{S}(s) & =\frac{\beta}{\Gamma\left(\alpha_{\text {eff }}\right)} \cdot(\beta . s)^{\alpha_{\text {eff }}-1} \exp (-\beta . s) .
\end{aligned}
$$

Unfortunately, the assumed independence of neighboring scatterers does not represent the actual macro-relief spatial correlation.

One way to account for spatial dependence is to assume a correlation coefficient $\rho_{\sigma}$ between the neighboring cross sections [15]. If $\sigma\left(\vec{r}_{i}\right)=\left\|a_{i}^{2}\right\|=\left\|a^{2}\left(\vec{r}_{i}\right)\right\|$ defines the elementary cross section for the scatterers located in $\vec{r}_{i}$ and if it is assumed that the neighboring cross sections located in $\vec{r}_{1}$ and $\vec{r}_{2}$ have the same mean $\left\langle\sigma\left(\vec{r}_{1}\right)\right\rangle$ and same variance $\left\langle\left(\sigma\left(\vec{r}_{1}\right)-\left\langle\sigma\left(\vec{r}_{1}\right)\right\rangle\right)^{2}\right\rangle$, then for a stationary process, the correlation coefficient becomes

with

$$
\rho_{\sigma}(r)=\frac{\left\langle\sigma\left(\vec{r}_{1}\right) \sigma\left(\vec{r}_{1}+\vec{r}\right)\right\rangle-\left\langle\sigma\left(\vec{r}_{1}\right)\right\rangle^{2}}{\left\langle\sigma\left(\vec{r}_{1}\right)^{2}\right\rangle-\left\langle\sigma\left(\vec{r}_{1}\right)\right\rangle^{2}}
$$

$$
r=\left\|\vec{r}_{2}-\vec{r}_{1}\right\| .
$$

The scatterers are now located on a regularly spaced dense array.

For very small ensonified areas, the cross section $\sigma\left(\vec{r}_{i}\right)$ must be $\Gamma$-distributed with a shape factor $\alpha$ such that $\operatorname{var}\left(\sigma\left(\vec{r}_{i}\right)\right) /\left\langle\sigma\left(\vec{r}_{i}\right)\right\rangle^{2}=1 / \alpha$. In this fully correlated scheme, amplitudes $\left\{a_{i}\right\}$ are constant over the resolution cell, and (10) leads to

$$
Y\left(\vec{r}_{i}\right)=\left\|\left(a\left(\vec{r}_{i}\right)\right)\right\|^{2} \cdot Z=\sigma\left(\vec{r}_{i}\right) \cdot Z\left(\vec{r}_{i}\right) .
$$

In this special case, $\alpha_{\text {eff }}=\alpha, S=\sigma$, and the backscattering energy $Y$ distribution is still given by (13). With the noncorrelated speckle, the $Y$ correlation coefficient becomes

$$
\rho_{Y}(r)=\rho_{S}(r) \frac{\left\langle S^{2}\right\rangle-\langle S\rangle^{2}}{2\left\langle S^{2}\right\rangle-\langle S\rangle^{2}}=\frac{\rho_{S}(r)}{\alpha_{\mathrm{eff}}+2}=\frac{\rho_{\sigma}(r)}{\alpha+2} .
$$

Even for this ideal case, the speckle term induces a decorrelation between the received energy $Y$ and the seafloor-dependent cross section $\sigma$.

When averaging, the noise speckle term vanishes. By definition, the average backscatter energy from scatterers located at $\vec{r}_{i}$ is the backscattering strength $B S$. As the ensonified area is small, the local cross section is assumed constant.

Equation (16) leads to a phenomenological interpretation of the reverberation model

$$
\left\langle Y\left(\vec{r}_{i}\right)\right\rangle=\sigma\left(\vec{r}_{i}\right)=B S\left(\theta_{\mathrm{inc}}\left(\vec{r}_{i}\right)\right) .
$$

Equation (18) links the cross section $\sigma$ to $B S$.

Firstly, the cross section depends on the seafloor geoacoustical characteristics since the backscattering strength $B S$ is a function of the seafloor acoustical impedance and its microroughness spectrum (with respect to the ensonified area size) [7]. Secondly, as the incidence angle $\theta_{\text {inc }}\left(\vec{r}_{i}\right)$ between two consecutive soundings is set by the macro-relief local slope ([AUTHOR: FIG. 9 IS CITED OUT OF ORDER. PLEASE CITE FIGS. 7 AND 8 FIRST.-ED.]Fig. 9), (18) also underlines the effect of the macro-relief spectrum on the cross section.

On a larger ensonified area, the resolution cell can no longer precisely describe the macro-relief variations and an averaging process occurs. The $Y$ statistical distribution can still be considered as a $K$-distribution with an adapted shape factor, $\alpha_{\text {eff }}$, and a correlation coefficient, $\rho_{Y}$. For demonstration, suppose a one-dimensional (1-D) seafloor characterized by its cross section $\sigma$ which is exponentially correlated and $\Gamma$-distributed with a constant shape factor $\alpha$

$$
\rho_{\sigma}(r)=\exp \left(-\frac{|r|}{l_{c}}\right)
$$

where $l_{c}$ is for the correlation length of the macro-relief, $l_{a}$ the ensonified area size, and $\gamma=l_{a} / l_{c}$. Then, [15]

$$
\begin{aligned}
\alpha_{\mathrm{eff}} & =\alpha \cdot \frac{\gamma^{2}}{2(\exp (-\gamma)+\gamma-1)} \\
\rho_{Y}(r) & =\rho_{\sigma}(r) \cdot \frac{(\exp (\gamma / 2)-\exp (-\gamma / 2))^{2}}{\gamma^{2} \cdot \alpha+4(\exp (-\gamma)+\gamma-1)} .
\end{aligned}
$$

When the ensonified area size increases, the shape factor $\alpha_{\text {eff }}$ of the measured energy $Y$ increases nearly linearly $\left(\alpha_{\text {eff }} \approx\right.$ $\gamma . \alpha / 2$ ), and the $K$-distribution tends to an exponential distribution as in a Rayleigh reverberation process (Fig. 7).

With a high-resolution MBES [3], $\rho_{Y}(r)$ is measured when $r$ is a multiple of $l_{a}$. In Fig. 8, the comparison of $\rho_{Y}(r)$ and $\rho_{\sigma}(r)$ reveals, first, the decorrelation effect due to the speckle term, and, second, the averaging effect on the ensonified area. The correlation coefficient of the first bin (circles) decreases because the growing ensonified area tends to remove the influence of the seafloor correlation. 


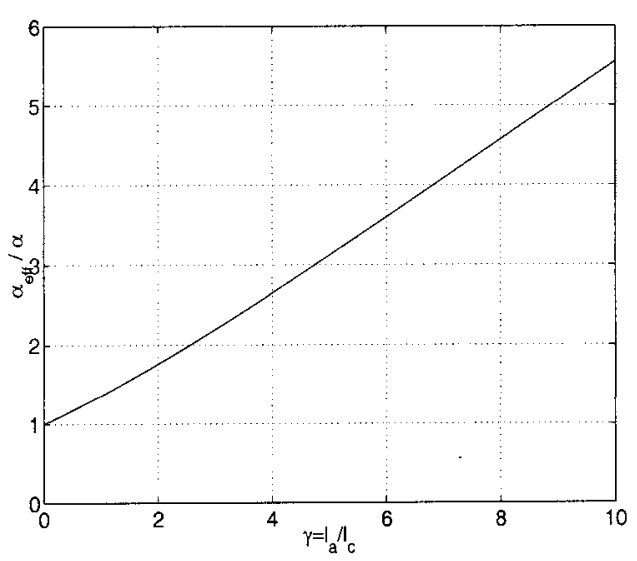

Fig. 7. The shape factor $\alpha_{\text {eff }}$ variation.

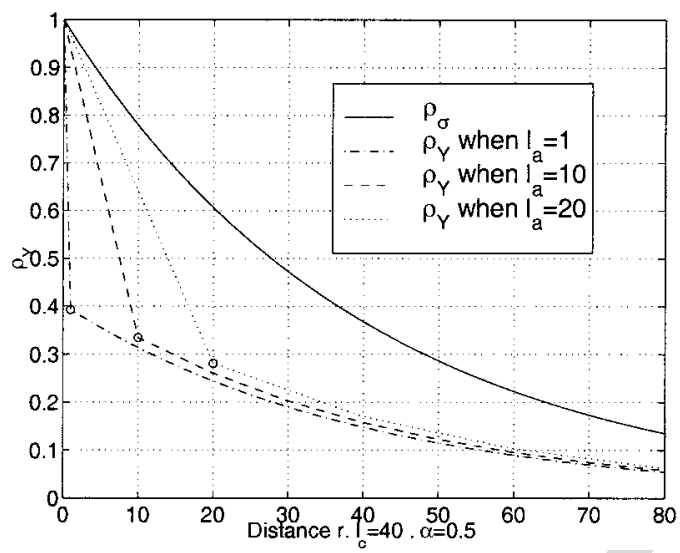

Fig. 8. The correlation coefficient $\rho_{Y}$ variation.

a) Incidence Angle Effects: The MBES transmission angle $\theta$ ranges from $0^{\circ}$ (vertical) to $75^{\circ}$ (high incidence angles). These transmitting angle changes create variations of both the local incidence angle $\theta_{\text {inc }}\left(\vec{r}_{i}\right)$ (Fig. 9) and the ensonified area $A\left(\theta_{\text {inc }}\left(\vec{r}_{i}\right)\right)$.

Equation (20) already accounts for the $Y$ statistical distribution and its shape factor $\alpha_{\text {eff }}$ changes with the ensonified area given in Fig. 10. This section also demonstrates the dependence of $\alpha=\alpha\left(\bar{\theta}_{\text {inc }}\right)$ on the average incidence angle.

The local incidence angle $\theta_{\text {inc }}\left(\vec{r}_{i}\right)$ depends on the macrorelief local slope $\phi\left(\vec{r}_{i}\right)$ and on the transmission angle $\theta$ (Fig. 9)

$$
\theta_{\text {inc }}\left(\vec{r}_{i}\right)=\theta-\phi\left(\vec{r}_{i}\right)
$$

Unfortunately, the local slopes $\phi\left(\vec{r}_{i}\right)$ between two consecutive soundings are not available. The DTM only provides an average local slope $\theta_{D T M}$. Therefore, the local slope $\phi\left(\vec{r}_{i}\right)$ is then considered as a random variable. Its variance depends both on the macro-relief surface properties and on the spacing between two soundings [16]. Equation (22) induces a similar statistical behavior for the local incidence angles $\theta_{\text {inc }}\left(\vec{r}_{i}\right)$ centered on the average incidence angle

$$
\bar{\theta}_{\text {inc }}=\left\langle\theta_{\text {inc }}\left(\vec{r}_{i}\right)\right\rangle=\theta-\theta_{\mathrm{DTM}} .
$$

For convenience, let $\theta_{\mathrm{DTM}}=0$. In the case of a small ensonified area, $S\left(\vec{r}_{i}\right)=\sigma\left(\vec{r}_{i}\right)=B S\left(\theta_{\text {inc }}\left(\vec{r}_{i}\right)\right)$. We make the hypothesis of a Gaussian-distributed local slope, which, from

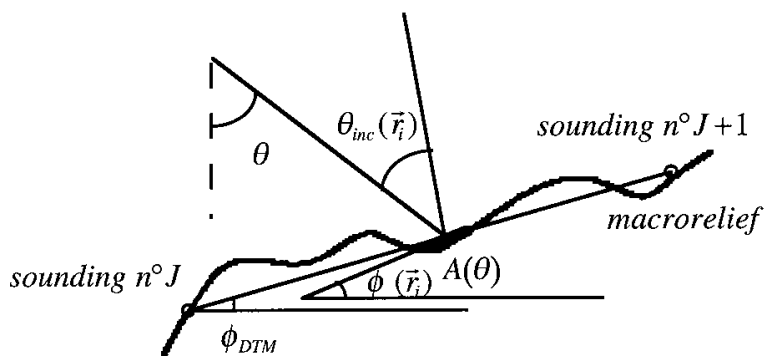

Fig. 9. Transmission, incidence, local slope angles, ensonified area, and DTM angle.

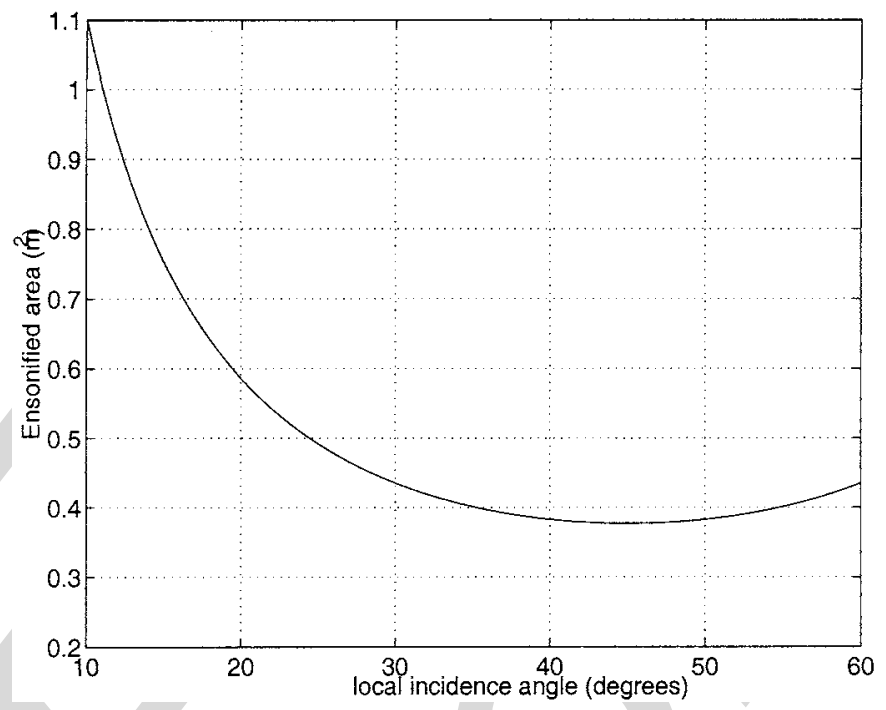

Fig. 10. Local incidence angle versus ensonified area.

(22), also implies a Gaussian distribution for $\theta_{\text {inc }}\left(\vec{r}_{i}\right)$ with mean $\theta$ and with constant standard deviation across-track. The variance and mean of $B S\left(\theta_{\text {inc }}\left(\vec{r}_{i}\right)\right)$ are computed to find $1 /(\alpha \theta)=$ $\left(\left\langle\sigma^{2}\right\rangle /\langle\sigma\rangle^{2}\right)-1$. The probability density of $S\left(\vec{r}_{i}\right)$ can be expressed by

$$
p_{S}\left(B S\left(\theta_{\text {inc }}\right)\right)=\frac{p_{\theta_{\text {inc }}}\left(\theta_{\text {inc }}\right)}{\left|\frac{d B S\left(\theta_{\text {inc }}\right)}{d\left(\theta_{\text {inc }}\right)}\right|} .
$$

For the simulation depicted in Fig. 11, we use the typical backscattering strength $B S(\theta)$ [Fig. 11(a)] defined by (6).

Finally, (20) includes the ensonified area effects to assess the $Y$ shape factor $\alpha_{\text {eff }}$. Fig. 11(b) shows the calculated $1 / \alpha_{\text {eff }}$ variations.

$\alpha_{\text {eff }}$ shows higher values at intermediate incidence angles than at low or high incidence angles, and these values are dependent on the macrorelief slope variance. In fact, the ensonified area size increases at low and high incidence angles (Fig. 10), which would tend to decrease the normalized variance, but the corresponding backscattering curve slope $d B S\left(\theta_{\text {inc }}\right) / d \theta_{\text {inc }}$ is larger for these values, creating deep modulations of the cross section and increasing the normalized variance: this last effect is the one that finally dominates. These simulations and this nonRayleigh correlated reverberation model underline and quantify the difference between the seafloor-dependent cross section $\sigma$ and the energy $Y$. 


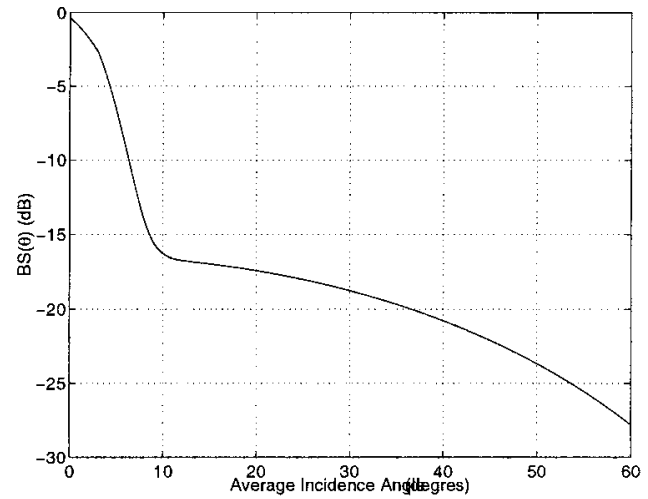

(a)

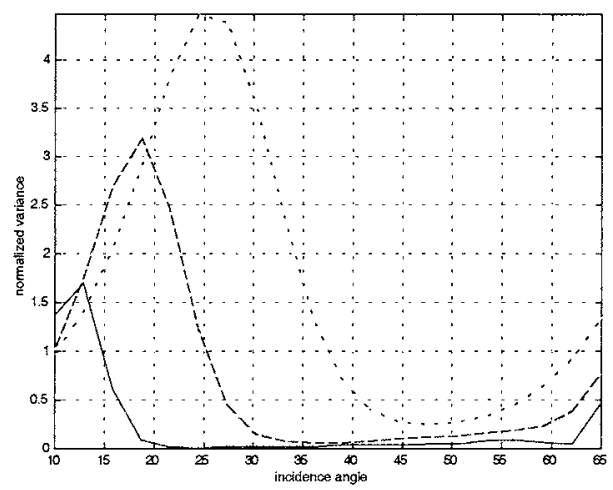

(b)

Fig. 11. (a) Simulated backscattering strength. (b) Normalized variance $1 / \alpha_{\text {eff }}$ with the standard deviation of the slope distribution. (dotted line: $\sigma_{\text {slope }}=10^{\circ}$, dashed line: $\sigma_{\text {slope }}=7^{\circ}$, continuous line: $\sigma_{\text {slope }}=4^{\circ}$ ).

In the next section, measured MBES data are processed and demonstrate the validity of this non-Rayleigh model for highresolution seafloor backscatter.

\section{Measured MBES Backscatter Data Analysis}

1) Measured Particularities: The following MBES data were recorded on the French inner shelf (15-60 m deep) of the Atlantic using Simrad EM 1000 [3]. For high incidence angles, two consecutive pixels of the backscatter image are separated by $15 \mathrm{~cm}$. The echo energy samples are recorded between $-64 \mathrm{~dB}$ and $+64 \mathrm{~dB}$ in $0.5-\mathrm{dB}$ steps. The available values for energy $Y$ are $\left\{y_{k}=y_{\min } 10^{a . k}, 0 \leq k \leq 255\right\}$ with $y_{\min }=10^{-6.4}$ and $a=0.05$. This logarithmic sampling transforms the continuous statistical distribution of $Y, p_{Y}(y)$ into a discrete probability law $p_{Y}^{S}\left(y_{k}\right)$. Because of the analytical complexity, a first-order approximation is used to derive the discrete probability from the continuous density

$$
p_{Y}^{S}\left(y_{k}\right)=\frac{(a-1) y_{k} p_{Y}\left(y_{k}\right)}{\sum_{k=0}^{255}(a-1) y_{k} p_{Y}\left(y_{k}\right)} .
$$

In order to focus only on statistical disparities, MBES data are normalized. On each partial subarea, the corresponding $B S$ is calculated by averaging data coming from the different pings. Next, the computed $B S$ value is subtracted from all the sonar data. The resulting sonar image exhibits no mean variations across swath.
2) K-Distribution Fitting Measured Data Histograms: In this section, the average incidence angle equals $60^{\circ}$. Fig. 12 shows partial backscatter images recorded on three different seafloor areas (rock, gravel, and sand). The corresponding energy $Y$ histogram is also depicted with the fitting results of a (discrete) $K$-distribution and a (discrete) exponential distribution. The estimated $K$-distribution shape factor $\hat{\alpha}_{\text {eff }}$ is calculated for each seafloor section according to the moment method with

$$
\hat{\alpha}_{\mathrm{eff}}=\frac{1}{\frac{\left\langle Y^{2}\right\rangle_{\text {nat }}}{2\langle Y\rangle_{\text {nat }}^{2}}-1}
$$

and $\hat{\beta}=\hat{\alpha}_{\text {eff }} /\langle Y\rangle_{\text {nat }}$. Additional fitting tests were carried out especially with $\Gamma$-distributions and general laws deriving from the Pearson system [17]. To assess the accuracy of the law fitting, the nonparametric Kolmogorov-Smirnoff test [18] was calculated. For each measured data $Y$ histogram, the corresponding measured data cumulative distribution function (CDF), $\hat{F}(x)$, is estimated and compared to the theoretical CDFs $F(x)$, thanks to the computation of the Kolmogorov $L_{1}$-distance, $D_{k}=\sup |F(x)-\hat{F}(x)|$. After setting a confidence threshold $\beta=0.05$ and taking into account the measured number of data $M$, an acceptance distance $D_{\text {acc }}$ is calculated. If $D_{k}$ stands below $D_{\text {acc }}$, the theoretical distribution may be accepted to describe the measured data with a $1-\beta$ confidence level. Table II shows the Kolmogorov and acceptance distances for the theoretical distributions.

The estimated shape factor $\hat{\alpha}_{\text {eff }}$ for rock, gravel, and sand seafloors is $0.9,2.7$, and 6.8 , respectively. The ensonified area is small enough to make the relief variations visible on the textured backscatter image (rocky area). As predicted, the tail of the measured histogram is too long to be described by the Rayleigh law. In the case of the gravel and silt seafloor, the $K$-distribution test distance is very close to the acceptance distance, which means that the distribution fits. In the case of a rock seafloor, the test fails. However, it is not sufficient to remove the hypothesis, since the Smirnov-Kolmogorov test suffers from two principal defects: sensitivity to the maximum values of the histogram and bad behavior when the distribution parameters are estimated. Nevertheless, when the relief is not deeply modulated (sandy area), no energy change appears on the nontextured image. Hence, the $K$-distribution is close to the Rayleigh distribution. The shape factor variation succeeds in describing the image textures and the changes in the nature of the ensonified seafloor. Apart from the mean backscattering strength, the statistical distribution provides additional information to improve the seafloor characterization techniques. When handling large amounts of measured data (Table II), the Kolmogorov test is too severe and not fulfilled by any theoretical distributions even by laws deriving from the Pearson system. For a large set of backscatter images, $K$-distributions challenge the Pearson system laws and are more robust to noise during the parameter estimation step.

3) Incidence Angle Effects: For the three seafloor areas, the estimated shape factor $\hat{\alpha}_{\text {eff }}$ of the energy $Y$ was computed for 11 different average incidence angle $\theta_{\text {inc }}$ sectors, each sector being 5 degrees wide. The average value of the first incidence 

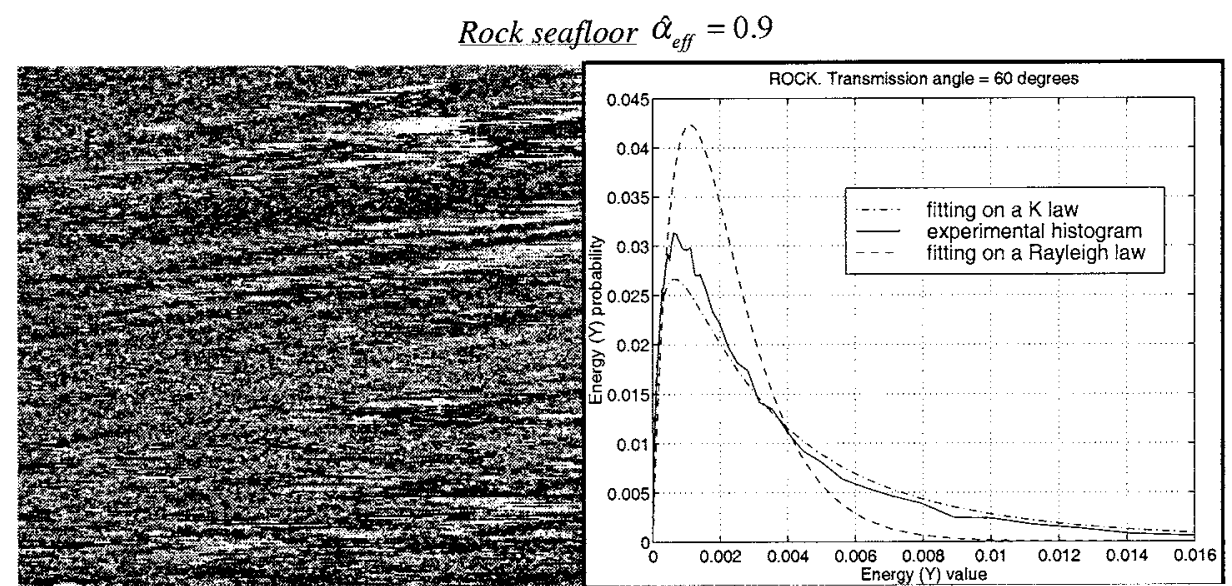

\section{Gravel seafloor $\hat{\alpha}_{\text {eff }}=2.7$}
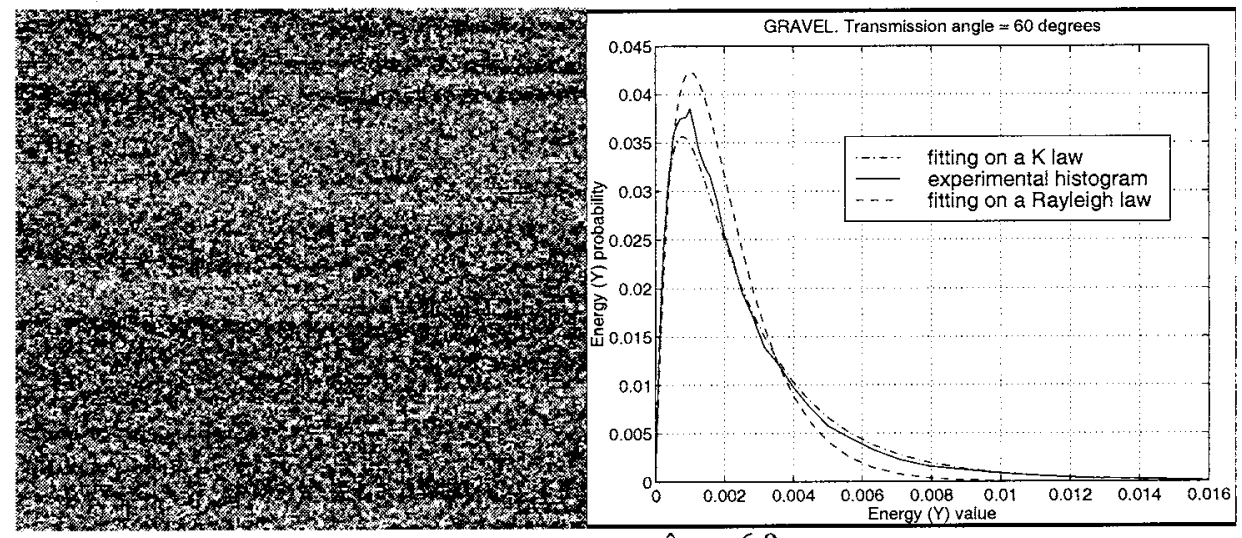

Sand seafloor $\hat{\alpha}_{e f f}=6.8$
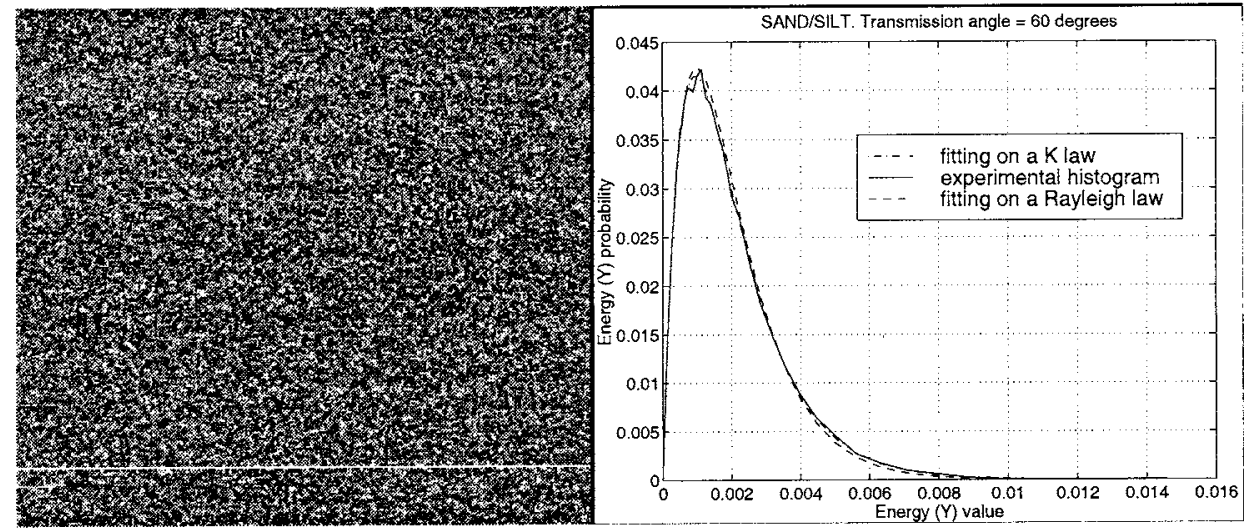

Fig. 12. Measured histograms and statistical distribution fitting.

TABLE II

Statistical Distribution FitTing MBES Measured Data and KOLMOGOROV DISTANCE

\begin{tabular}{l|l|l|l|l}
\hline$\theta$ & $12.3^{\circ}$ & $15.7^{\circ}$ & $30^{\circ}$ & $60^{\circ}$ \\
\hline$\theta-\theta_{0}$ & $1.37^{\circ}$ & $1.02^{\circ}$ & $0.43^{\circ}$ & $0.08^{\circ}$ \\
\hline
\end{tabular}

angle sector is $12.5^{\circ}$ and equals $62.5^{\circ}$ for the last. Fig. 13 shows evolution of the $Y$ statistical distribution (via the inverse of the shape parameter) according to the average incidence angle, especially for a rough seafloor. The observed tendencies confirm the non-Rayleigh correlated reverberation model [Fig. 11(b)]: the parameter $\alpha_{\text {eff }}$ decreases with low and high incidence angles for rough seafloors. These variations are smaller when the roughness decreases as in the gravel and silt case.

4) The Data Averaging Effect: For larger scale representations, the backscatter image pixel size may exceed the MBES resolution $(0.15 \mathrm{~m})$. During the image construction, $N_{\text {av }}$ raw data are averaged to figure out the pixel reflectivity $\bar{Y}^{N_{\mathrm{av}}}$

$$
\bar{Y}^{N_{\mathrm{av}}}=\frac{1}{N_{\mathrm{av}}} \sum_{k=1}^{N_{\mathrm{av}}} Y(k) .
$$




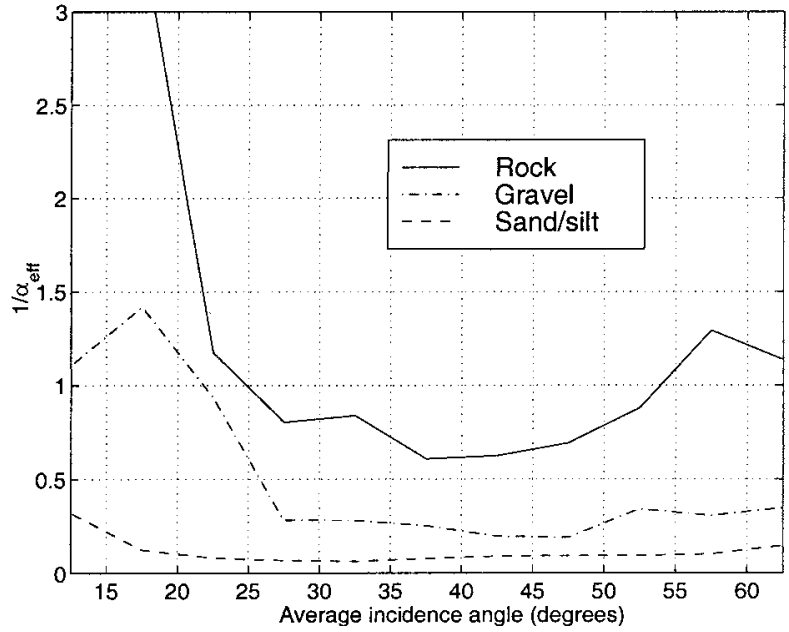

Fig. 13. Shape factor $1 / \alpha_{\text {eff }}$ variations with the average incidence angle.

Referring to the product model in (14), (25) is equivalent to

$$
\bar{Y}^{N_{\mathrm{av}}}=\frac{1}{N_{\mathrm{av}}} \sum_{k=1}^{N_{\mathrm{av}}} S(k) Z(k) .
$$

In a Rayleigh reverberation scheme, the local reflectivity $S$ is constant over the resolution cell. Then

$$
\bar{Y}^{N_{\mathrm{av}}}=S \cdot\left(\frac{1}{N_{\mathrm{av}}} \sum_{k=1}^{N_{\mathrm{av}}} Z(k)\right)=S \cdot \bar{Z}^{N_{\mathrm{av}}} .
$$

The speckle term $\bar{Z}^{N_{\mathrm{av}}}$ is $\Gamma$-distributed with a shape parameter $\alpha_{\text {eff }}=N_{\text {moy }}$ (equivalent to a $\chi^{2}$-law with a $2 N_{\text {moy }}$ axis of freedom). The speckle noise effect is slowly removed when averaging and the local reflectivity $S$ (signal) influence is enhanced. The derivation of (26) for non-Rayleigh reverberation schemes is no longer direct. Assuming that $N_{a v} * 0.15$ remains small compared to the macro-relief correlation length, (27) is still valid and induces the following "Generalized $K$-Law" statistical distribution for the pixel reflectivity $\bar{Y}^{N_{\mathrm{av}}}$ :

$$
\begin{aligned}
& p_{Y^{N_{\mathrm{av}}}}(y)=\frac{1}{\Gamma\left(\alpha_{\mathrm{eff}}\right) \Gamma\left(N_{\mathrm{av}}\right)} \frac{2}{y}\left(\beta \cdot N_{\mathrm{av}} \cdot y\right)^{\left(\alpha_{\mathrm{eff}+N_{\mathrm{av}}}\right) / 2} \\
& \cdot K_{\alpha_{\text {eff }-\mathrm{N}_{\mathrm{av}}}}\left(2 \sqrt{N_{\mathrm{av}} \cdot \beta \cdot y}\right) \\
& \alpha_{\text {eff }}=\frac{1}{\frac{N_{\mathrm{av}}}{N_{\mathrm{av}}+1}} \frac{\left\langle Y^{2}\right\rangle}{\langle Y\rangle^{2}}-1 \text {. }
\end{aligned}
$$

This generalized $K$-distribution derives from the product of two independent $\Gamma$-distributed random variables. MacDaniel [14] used it to characterize the seafloor reverberation process when taking into account the transmitted waveform and beamforming. Expressions for the law parameter estimation are derived in [19]. However, for the backscatter image, $N_{\mathrm{av}}$ is identified. We use equation (29) to estimate the extra parameter $\hat{\alpha}_{\text {eff }}$.

In Fig. 14, a generalized $K$-distribution related to the non-Rayleigh reverberation, and a $\chi^{2}$-law related to the Rayleigh scheme, are fitted to MBES measured data recorded on a rocky seabed. $N_{\mathrm{av}}$ is set to 16 , corresponding to an image pixel size of $2.4 \mathrm{~m}$. The generalized $K$-distribution is adapted to the data and fulfills the Kolmogorov-Smirnov test. In spite

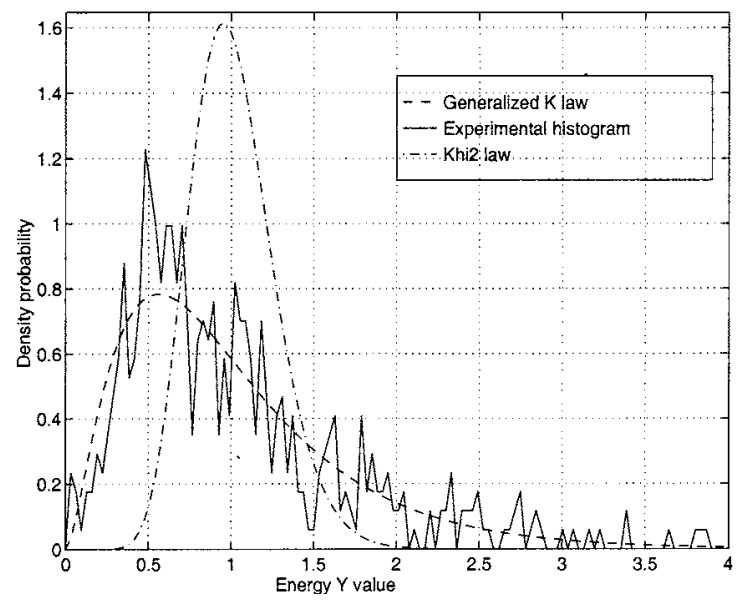

Fig. 14. Measured histogram for averaged MBES data. $N_{\text {av }}=16$. Rock seafloor. Transmission angle $=60^{\circ} . \hat{\alpha}_{\text {eff }}=2.6$.

of data averaging, the measured data statistical distributions do not tend toward the $\chi^{2}$-law but remain much spikier with strong asymmetry around the average value. On this particular data set and seafloor area, the averaging data process seems to reduce speckle noise without eliminating the seafloor characteristics.

\section{CONCLUSION}

Seafloor characterization using sonar images is currently strongly focused on the use of multibeam echosounders, which nowadays reach a high level of performance. In order to take full advantage of these systems' capabilities, the recorded data must first be normalized and cleared of the various artifacts produced by the sensor, both because of its physical characteristics (transducer sensitivity and directivity) and because of the unavoidable approximations introduced in the real-time processing software. In particular, this step is indispensable prior to a relevant analysis of the backscattered signal amplitude.

As shown on many seafloor sonar images, MBES beam patterns may strongly modulate and corrupt the recorded backscatter image. The correction procedure proposed here aims at compensating data coming from individual beams, accounting for the antenna sensitivity and beam pattern, and correcting possible inaccuracies in the ensonified footprint estimation. This has to be done as far as possible using MBES characteristics obtained from the constructor; when this is not possible or when the characteristics are dubious, it is proposed to estimate them directly from an identification with measured data obtained on flat homogeneous zones, using an appropriate model described above. The correction method typically leaves uncorrected artifacts of the order of 2-3 dB at oblique incidences (in favorable cases, results better than $1 \mathrm{~dB}$ were obtained). However, for steep angles, the signal instability associated with insufficient sampling makes the method less reliable. In addition, echo signal simulations demonstrate a strong averaging effect inside the ensonified area of these near-nadir beams that can hardly be compensated practically.

With low-frequency MBES, the averaged backscattered intensity is the only discriminating feature usable for seafloor characterization purposes [2]. However, the good resolution associated with high-frequency MBES highlights differences 
in the statistical behaviors observed on images recorded on various seafloor types. In particular, rough seafloors with strong local slope variations induce strong modulations on the recorded backscattering strength; the associated statistical distribution tends to be spiky and no longer fits the classical Rayleigh distribution. The $K$-distribution proves to be better adapted to various measured statistical distributions; apart from the average value, the shape factor provides an efficient feature usable for discriminating the nature of the seafloor. On the other hand, MBES geometry makes it possible to collect echoes associated with various footprint sizes and incidence angles. The ensonified footprint area is maximal at near-specular angles (where the pulse-duration limitation is ineffective) and at high incidence angles (due to the increase in the footprint alongtrack dimension). Classical analyses predict that the averaging effect inside these large ensonified patches (featuring a high number of scatterers) leads to statistical distributions closer to Rayleigh's than for data obtained from moderate oblique angles; however, the measured analysis does not verify this forecast. A reverberation statistical model is introduced, explicitly taking into account the correlation properties of the seafloor roughness and the echosounder geometrical configuration. Providing a satisfactory agreement with the measured data behavior, this model makes clear the effect of the angular backscattering strength first derivative on statistical distributions near the nadir and at high incidence angles. Indeed, the amplitude statistical analysis, the backscattering strength estimation, and the echo signal modeling are closely interdependent.

The introduction of this non-Rayleigh distribution in the backscattered signal model allows us to improve the data analysis for seafloor characterization and, in particular, to take into account the geometry effects of the sounder.

\section{ACKNOWLEDGMENT}

The authors wish to thank J. M. Augustin (IFREMER, France) for his help during sonar data interpretation and handling. They also wish to thank J. V. Gardner (U.S. Geological Survey, California) for his advice while writing this paper.

\section{REFERENCES}

[1] Simrad, "Simrad EM12. Hydrographic echo sounder. Product description," Simrad subsea A/S, Horten, Norway, 1992.

[2] S. Dugelay et al., "A new method for seafloor characterization with multibeam echosounders: Image segmentation using angular backscatter," in Proc. 3rd Eur. Conf. Underwater Acoustics, vol. I, 1996, pp. 439-444.

[3] Simrad, "Simrad EM1000. Hydrographic echo sounder. Product description," Simrad subsea A/S, Horten, Norway, 1992.

[4] L. Hellequin, "Statistical characterization of multibeam echo sounder data," in Proc. OCEANS'98 MTS/IEEE Conf., vol. 1, 1998, pp. 228-233.

[5] A. P. Lyons and D. A. Abraham, "Statistical characterization of high frequency shallow-water seafloor backscatter," J. Acoust. Soc. Amer., pt. 1, vol. 106, no. 3, pp. 1307-1315, 1999.

[6] C. Augris et al., "Le plateau continental basque. Caractérization des fonds marins à l'aide d'un sondeur multifaisceaux,", Internal rep. IFREMER, 1998

[7] X. Lurton et al., "Shallow-water seafloor characterization for high-frequency multibeam echosounder: Image segmentation using angular backscatter," in Proc. High Frequency Shallow Water Acoustics, SACLANTCEN Conf., N. Pace, Ed. La Spezia, Italy, 1997.
[8] L. Hellequin, J. M. Augustin, and X. Lurton, "Postprocessing and signal corrections for multibeam echosounder images," in Proc. OCEANS'97 MTS/IEEE Conf., vol. 1, 1997, pp. 23-26.

[9] L. Brekhovskikh and Y. P. Lysanov, Fundamentals of Ocean Acoustics. Heidelberg, Germany: Springer-Verlag, 1982.

[10] V. V. Olcheskii, Characteristics of Seafloor Reverberation. New York: Plenum, 1967.

[11] K. D. Ward, "Compound representation of high resolution sea clutter," Electron. Lett., vol. 17, no. 16, pp. 561-563, 1981.

[12] E. Jakeman, "Non-Gaussian models for the statistics of the scattered waves," Advances in Physics, vol. 37, no. 5, pp. 471-529, 1988.

[13] N. L. Johnson, Distribution in Statistics: Continuous Univariate Distribution. New York: Wiley, 1969, vol. I.

[14] S. T. MacDaniel, "Seafloor reverberation fluctuations," J. Acoust. Soc. Amer, vol. 88, pp. 1530-1535, 1990.

[15] C. J. Oliver, "Correlated $K$-distributed clutter models," Optica Acta, vol. 32, no. 12, pp. 1515-1547, 1985.

[16] J. Dunlop, "Statistical modeling of sidescan sonar images," in Proc. OCEANS'97 MTS/IEEE Conf., 1997, pp. 33-38.

[17] L. Hellequin, "Analyze statistique et spectrale des signaux de sondeurs multifaisceaux EM950. Application à l'identification des fonds sousmarins,", Thèse de l'Université de Rennes 1, 1998.

[18] [AUTHOR: PLEASE PROVIDE THE LOCATION OF THE PUBLISHER.-ED.]P. J. Bickel and K. A. Doksum, Mathematical Statistics, Basic Ideas and Selected Topics: Holden-Day, 1977.

[19] M. Gu and D. A. Abraham, "Parameter estimation for McDaniel's non Rayleigh reverberation model," in Proc. Oceans'99 MTS/IEEE Conf., vol. 1, 1999, pp. 279-283.

Laurent Hellequin was born in 1971. He received the degree in electronic engineering from the Institut National Polytechnique de Grenoble (INPG), Grenoble, France, and the Ph.D. degree. His doctoral work focused on subjects related to sonar signal processing and seafloor characterization.

While completing his doctoral work, he worked at both Telecom Bretagne, France and IFREMER, Plouzane, France. In 2000-2001, during a post-doctoral period conducted at the Center for Coastal and Ocean Mapping, University of New Hampshire, he focused on issues regarding quality of multibeam echosounder data.

Jean-Marc Boucher (M'xx) was born in 1952. He received the engineering degree in telecommunications from the "Ecole Nationale Supérieure des Telecommunications," Paris, France in 1975 and the "Habilitation à Diriger des Recherches" degree in 1995 from the University of Rennes 1, Rennes, France.

$\mathrm{He}$ is currently Professor in the Department of Signal and Communications, Ecole Nationale Superieure des Telecommunications de Bretagne, France. His research interests include estimation theory and classification, Markov models, blind deconvolution, wavelets, and multiscale image analysis, with applications to radar and sonar, seismic, and biological signals. He has published approximately 100 papers in these areas in international journals and conferences.

Xavier Lurton was born in Bordeaux, France, in 1955. He received the Ph.D. degree in applied acoustics from the Université du Maine, Le Mans, France, in 1979.

From 1981 to 1989, he was with Thomson-Sintra ASM, working in the field of underwater sound propagation modeling for naval applications. In 1989, he joined IFREMER, Plouzane, France, the French oceanological research agency. After working on various acoustical oceanography applications (ocean tomography, telemetry, fisheries sonar) and managing the Ifremer acoustics laboratory for five years, he is now in charge of a research program on seafloor characterization using multibeam echosounders. His current interests are both in the physics of seabed backscattering and in sonar engineering and signal processing. 